\title{
Evaluation of Acute Neuro Toxicity of CCRT with Temozolomide Versus RT Alone in Patients with High Grade Gliomas
}

Md. Mamun Or Rasheed ${ }^{1 *}$, Md. Zillur Rahman Bhuiyan², Md. Nazir Uddin Mollah², Muhammad Rafiqul Islam ${ }^{3}$, Md. Tariq $\operatorname{Hasan}^{4}$, Md. Mostafizur Rahman ${ }^{5}$

\footnotetext{
${ }^{1}$ Assistant Professor, Department of Oncology, Bangabandhu Sheikh Mujib Medical University, Dhaka, Bangladesh

${ }^{2}$ Associate Professor, Department of Oncology, Bangabandhu Sheikh Mujib Medical University, Dhaka, Bangladesh

${ }^{3}$ Assistant Professor, Department of Medical Oncology, National Institute of Cancer Research and Hospital, Dhaka, Bangladesh

${ }^{4} \mathrm{MD}$ (Resident) Department of Oncology, Bangabandhu Sheikh Mujib Medical University, Dhaka, Bangladesh

${ }^{5}$ Diploma in Anaesthesiology (Course), Sir Salimullah Medical College, Dhaka, Bangladesh
}

DOI: $10.36347 /$ sjams.2020.v08i06.006

| Received: 18.05.2020 | Accepted: 26.05.2020 | Published: 10.06.2020

*Corresponding author: Md. Mamun Or Rasheed

To study the survival outcome and toxicity grades in patients of high-grade glioma (HGG) treated with conformal radiation and temozolamide. High grade gliomas (HGG) are the most common primary CNS tumors in adult. Even with multidisciplinary approach the outcome is miserable. However recently concomitant chemoradiation (CCRT) with temozolomide has been effectively used. It has found to increase median survival with good clinical outcome in patients with malignant gliomas. This was a quasi experimental study done in the department of radiation oncology in National Institute of Cancer Research \& Hospital (NICRH) Mohakhali, Dhaka during January 2014 to December 2014. Patients with newly diagnosed, histologically proved high grade (WHO grade III \& grade IV) were assigned to receive radiation therapy alone (fractionated focal irradiation in daily fractions of 2 Gy in five fractions per week for six weeks, for a total $60 \mathrm{~Gy})$ or radiation therapy plus temozolomide $\left(75 \mathrm{mg} / \mathrm{m}^{2}\right.$ from $1^{\text {st }}$ day of radiation therapy to last day of radiation therapy), followed by six cycles of adjuvant temozolomide (150 to 200mg per square meter for five days during each 28 day cycle). The primary end point was haematological, neurotoxicities \& survival. A total sixty patients were enrolled for study, acute neurotoxicities toxicities were compared between the patiens of two arms. Patients treated by CCRT with temozolomide showed more toxicities than the radiation therapy alone in some stages. As a whole the differences were not statistically significant.

Keywords: High grade gliomas, concurrent chemoradiation, radiation therapy, temozolomide.

Copyright @ 2020: This is an open-access article distributed under the terms of the Creative Commons Attribution license which permits unrestricted use, distribution, and reproduction in any medium for non-commercial use (NonCommercial, or CC-BY-NC) provided the original author and source are credited.

\section{INTRODUCTION}

Primary brain tumors comprise only approximately 2 percent of all the malignant diseases [1]. However, the major data source includes the Surveillance, Epidemiology and End Results (SEER) reported with an incidence of 6.5 per 100000 persons, more than 17000 cases are diagnosed every year in the united states, with approximately 13,000 associated deaths [2]. More specific CNS tumor types also differ in incidence rate based on anatomical location and also with age [3]. Gliomas constitute 40 percent of all primary CNS tumors [4]. Two third of gliomas are high grade, which comprise the glioblastoma multiforme (GBM), anaplastic astrocytoma (AA), anaplastic oligodendroglioma, anaplastic oligoastrocytoma and less common varieties such as anaplastic ependymoma and anaplastic ganglioglioma [5]. Male to female ratio among affected patient is about 3:2 and most of the
HGG are sporadic, although they are associated with genetic syndromes $[6,7]$.

The peak age of onset for anaplastic astrocytoma is during the 4th and 5th decade, while GBM generally presented in the 6th and 7th decade [8]. There are several presumed reasons for miserable outcome of high-grade gliomas. First the tumor cells in GBM extensively infiltrate the surrounding brain parenchyma, thereby limiting the overall utility of surgical resection. Second the blood brain barrier is an obstacle to the adequately delivery of chemotherapy agents to brain tumors [9]. Third HGG is resistant to most cytotoxic agents, the expression of MGMT promoter methylation is thought to be the major mechanism of the resistance [10]. Therefore surgical resections alone is the limitation in the treatment of HGG [11]. HGG have high morbidity and mortality rate, even with optimal, treatment median survival is 
only 12 to 15 months and 2 years survival rate in the range of only 8 to 12 percent for glioblastoma multiforme and 2 to 7 years for anaplastic astrocytoma [12].

Without any treatment the median survival is only 3 to 6 months from the time of diagnosis [13]. The standard management of HGG involve cytoreduction through surgical resection when feasible followed by radiation therapy with or without adjuvant chemotherapy [14]. Adjuvant radiation therapy helps to decrease local failure, to delay recurrence and ultimately prolong survival up to 12 months [15]. Most recently, the effectiveness of this concomitant chemotherapy with temozolomide has also been reported in many studies. The results of many trials demonstrated that concomitant radiation therapy plus continuous daily temozolomide therapy followed by additional cycles of the standard regimen of adjuvant temozolomide therapy is well tolerated and may prolong survival in patient with malignant glioma. Temozolomide is very easy to administer and safe to handle and also produced by our domestic pharmaceuticals.

\section{METHODS}

Patients age at and above 18 and below 70 years, histological confirmed HGG with have a UICC performance status of at and below 70 will be enrolled as study population. Prior to treatment proper evaluation of adequate hematological, renal and hepatic functions will done ie absolute neutrophil count > $15 / \mathrm{mL}$, platelet count $>100000 / \mathrm{mL}$, hemoglobin $>10$ $\mathrm{gm} / \mathrm{dl}$ serum creatinine and total serum bilirubin less than 1.5 times the upper limit of normal and aspartate aminotransferase less than twice the upper limit of normal. Poor general physical conditions will not be taken into account. Pretreatment evaluation of tumor for planning and outcome prediction, computed tomography $(\mathrm{CT})$, magnetic resonance imaging (MRI) will also be done. As per inclusion criteria patients those who are eligible for CCRT with temozolomide to receive oral TMZ using the protocol proposed by Stupp et al., in 2013 at a dose $75 \mathrm{mg} / \mathrm{m}^{2}$ per day for seven days per week from the first day of RTx until the last day of RTx but for no longer than 49 days. TMZ should be given in empty stomach 1 hour before RT or in the morning on days without RTx. After four weeks break the patients then receive further six cycles of adjuvant TMZ, according to the standard five days schedule, every 28 days. Patients were weekly followed up, the baseline examination included full blood counts and blood chemistry tests and a physical examination. Antiemetic prophylaxis was also given before the initial doses of concomitant temozolomide and was reqired during the adjuvant five days courses of temozolomide. Toxic effects were graded according to the National Cancer Institute Common Toxicity Criteria, version 2.0, with a score of 1 indicating mild adverse effects, a score of 2 moderate adverse effects, a score of 3 severe adverse effects and a score of 4 , life threatening adverse effects if needed.

\section{RESULTS}

Acute neuro toxicities were compared between the patients of two arms. Patients treated by CCRT with temozolomide showed more toxicities than the conventional radiation treatment alone in some stages. As a whole the differences were not significant.

Table-1

\begin{tabular}{|l|l|l|l|l|}
\hline \multirow{2}{*}{ Smoking } & \multicolumn{2}{|l|}{ Category of treatment } & \multirow{2}{*}{$\boldsymbol{*}$-value } \\
\cline { 2 - 3 } & Experimental (Arm A) & Conventional (Arm B) & & \\
\hline Yes & $16(55.2)$ & $8(26.7)$ & \multirow{2}{*}{3.96} & 0.035 \\
\hline No & $13(44.8)$ & $22(73.3)$ & & \\
\hline Total & $30(100.0)$ & $30(100.0)$ & & \\
\hline
\end{tabular}

Out of 60 patients 24 patients were smokers. In arm A more than 55\% (16/30) patients used to smoke. In arm B only $26.7 \%$ patients were smoker. Significant statistical difference among the two groups was noted $(\mathrm{p}<0.05)($ Table-1).
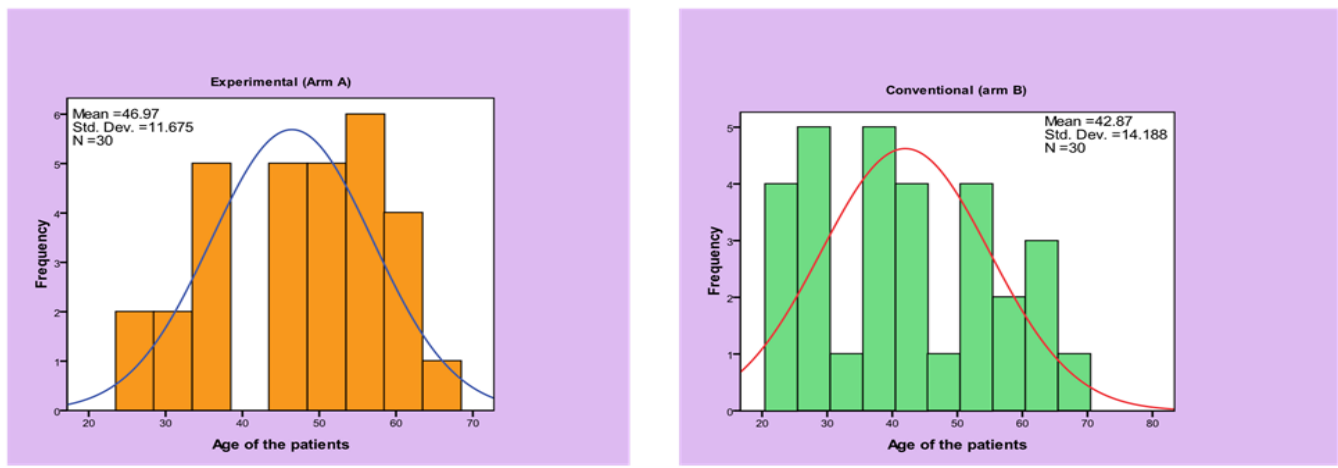

Fig-1: Age distribution of the patients 
Figure-1 shows the age distribution of the high grade gliomas patients. The mean age of the arm A patients was $46.9(\mathrm{SD} \pm 11.7)$ years and that of the arm
B patients was 42.8 ( $\mathrm{SD} \pm 14.2)$ years. No significant difference was observed between these two groups $(\mathrm{t}=-$ $1.22(\mathrm{df}=55.9) \mathrm{p}=0.227)$.

Table-2: Distribution of the patients by complaints

\begin{tabular}{|l|l|l|l|l|}
\hline \multirow{2}{*}{ Chief complains } & \multicolumn{2}{|c|}{ Experimental (Arm A) } & \multicolumn{2}{l|}{ Conventional (Arm B) } \\
\cline { 2 - 5 } & \multicolumn{2}{|c|}{ n } & (\%) & n \\
\hline Headache & 21 & 70.0 & 20 & 66.7 \\
\hline Vomiting & 14 & 46.7 & 11 & 36.7 \\
\hline Weakness & 11 & 36.7 & 12 & 40.0 \\
\hline Anaemia & 2 & 6.7 & 1 & 3.3 \\
\hline Clubbing & 2 & 6.7 & 1 & 3.3 \\
\hline Oedema & 2 & 6.7 & 0 & 0.0 \\
\hline
\end{tabular}

Table-2 shows the distribution of the patients by complaints. More than two third of the patients (70\%) in arm A complained about headache and in arm
B more than $66 \%$ (20/30) developed this symptoms. Vomiting was reported in $46.7 \%$ patients in arm A and $36.7 \%$ in arm $\mathrm{B}$.

Table-3: Distribution of the patients by histopathological findings

\begin{tabular}{|l|l|l|l|l|}
\hline \multirow{2}{*}{ Histopathological findings } & \multicolumn{2}{|l|}{ Category of the treatment } & \multirow{2}{*}{ Total } & \multirow{2}{*}{-value } \\
\cline { 2 - 4 } & Experimental (Arm A) & Conventional (arm B) & & \\
\hline Glioblastoma multiforme & $18(60.0)$ & $23(76.7)$ & $41(68.3)$ & 0.165 \\
\hline Anaplastic astrocytoma & $12(40.0)$ & $7(23.3)$ & $19(31.7)$ & \\
\hline Total & $30(100.0)$ & $30(100.0)$ & $60(100.0)$ & \\
\hline
\end{tabular}

Percentages are given in the parenthesis

Distribution of the patients by histopathological findings is shown in the above table (Table-3). More than 68\% (41/60)) patients were suffering from glioblastoma multiforme and the rest 19 patients $(31.7 \%)$ were suffering from anaplastic astrocytoma. Sixty percent of arm A patients and $76.7 \%$ of the arm B patients were the patients of glioblastoma multiforme. The percentages of patients of anaplastic astrocytoma in arm A \& B were 40 and 23.3 respectively. Difference of histopathological diagnosis across arms was not statistically significant $(\mathrm{p}>0.05)$.

Table-4: Distribution of the patients by Neurotoxicity

\begin{tabular}{|c|c|c|c|c|c|c|}
\hline \multicolumn{2}{|l|}{ Neurotoxicity } & \multicolumn{2}{|c|}{ Experimental (Arm A) } & \multicolumn{2}{|c|}{ Conventional (Arm B) } & \multirow[t]{2}{*}{$p$-value* } \\
\hline & & $\mathbf{N}$ & $\%$ & $\mathbf{n}$ & $\%$ & \\
\hline \multirow[t]{3}{*}{ At the beginning } & Grade 0 & 19 & 63.3 & 20 & 66.7 & \multirow[t]{3}{*}{1.000} \\
\hline & Grade 1 & 10 & 33.3 & 10 & 33.3 & \\
\hline & Grade 2 & 1 & 3.3 & 0 & 0.0 & \\
\hline \multirow[t]{3}{*}{7 days after treatment } & Grade 0 & 16 & 53.3 & 19 & 63.3 & \multirow[t]{3}{*}{0.689} \\
\hline & Grade 1 & 12 & 40.0 & 10 & 33.3 & \\
\hline & Grade 2 & 2 & 6.7 & 1 & 3.3 & \\
\hline \multirow[t]{3}{*}{14 days after treatment } & Grade 0 & 6 & 20.0 & 15 & 50.0 & \multirow[t]{3}{*}{0.046} \\
\hline & Grade 1 & 21 & 70.0 & 12 & 40.0 & \\
\hline & Grade 2 & 3 & 10.0 & 3 & 10.0 & \\
\hline \multirow[t]{4}{*}{21 days after treatment } & Grade 0 & 4 & 13.3 & 3 & 10.0 & \multirow[t]{4}{*}{0.640} \\
\hline & Grade 1 & 18 & 60.0 & 16 & 53.3 & \\
\hline & Grade 2 & 8 & 26.7 & 9 & 30.0 & \\
\hline & Grade 3 & 0 & 0.0 & 2 & 6.7 & \\
\hline \multirow[t]{4}{*}{28 days after treatment } & Grade 0 & 3 & 10.0 & 3 & 10.0 & \multirow[t]{4}{*}{0.162} \\
\hline & Grade 1 & 7 & 23.3 & 15 & 50.0 & \\
\hline & Grade 2 & 16 & 53.3 & 10 & 33.3 & \\
\hline & Grade 3 & 4 & 13.3 & 2 & 6.7 & \\
\hline
\end{tabular}

*Fisher's Exact test

In the above table (Table-4) neurotoxicity is compared in 5 different periods of time. At the beginning of radiation therapy 10 patients in both arms showed grade 1 neurotoxicity and 1 patient in arm B showed grade 2 neurotoxicity. Seven days after radiation 12 patients in arm $\mathrm{A}$ and 10 patients in arm B showed grade 1 neurotoxicity. At this stage 2 patient in arm A showed grade 2 neurotoxicity in comparison to 1 
patient in arm B. However, these differences were not statistically significant.

At day 14 of the treatment significant difference of neurotoxicity was observed across the two arms. In arm A, 21 patients suffered grade 1 neurotoxicity in contrast to 12 patients in arm B.

At day 21 of the treatment in arm A 18 had grade 1 neurotoxicity while this number in arm $\mathrm{B}$ was 16. At day 28 of the treatment more patients (15) in arm B suffered from grade 1 neurotoxicity while more patients (16) in arm A showed grade 2 neurotoxicity.

\section{DISCUSSION}

The mean age of the patients in arm $\mathrm{A}$ was 46.9 (SD \pm 11.7 ) years and that of the arm B was 42.8 $(\mathrm{SD} \pm 14.2)$ years. The age group distribution was almost identical which helped in minimizing bias. The other socio-demographic variables like socio-economic status, occupation or level education were not different across the two arms.

Regarding personal habits, out of 60 patients $24(40 \%)$ were smokers. This figure agrees with the national prevalence of smoking in Bangladesh. Significantly more patients in arm A (55\%) were smokers than arm B patients $(26.7 \%)$ which was purely incidental. However, no statistical significance were found between these two groups regarding betel leaf \& nut chewing habits $(p>0.05)$. Headache and vomiting were the two main presenting complaints of the patients in both arms. More than 68\% (41/60) patients were suffering from glioblastoma multiforme and the rest were suffering from anaplastic astrocytoma.

In about $87 \%$ cases craniotomy was performed. Stereotactic biopsy was done in $10 \%$ cases. The other modality was barhole biopsy. All of the patients in arm A tested positive for MGMT methylation test. Almost reverse findings was noted in arm B patients. Statistically this difference was highly significant $(\mathrm{p}<0.001)$

Neurotoxicity was compared in 5 different periods of time. At the beginning of radiation therapy 1 patient in arm B showed grade 2 neurotoxicity. Seven days after radiation 2 patients in arm A showed grade 2 neurotoxicity in comparison to 1 patient in arm $\mathrm{B}$. However, these differences were not statistically significant. At day 14 of the treatment significant difference of neurotoxicity was observed across the two arms $(\mathrm{p}<0.05)$; in arm A, 21 patients suffered grade 1 neurotoxicity in contrast to 12 patients in arm B. At day 21 of the treatment in arm A 18 had grade 1 neurotoxicity while this number in arm B was 16 . At day 28 of the treatment more patients (15) in arm B suffered from grade 1 neurotoxicity while more patients (16) in arm A showed grade 2 neurotoxicity. Twice the number of patients in arm A showed grade 3 toxicity than arm B patients (4 vs. 2). However, these differences were not significant statistically. Similar results were reported by Wen et al., and Sathornsumetee et al., [1, 16].

Acute neuro toxicity was also compared in several different periods of time starting from the beginning of the treatment to after 6 months of treatment completion. However, no differences of neuro toxicities were statistically significant between these two arms ( $p>0.05)$. This difference was statistically significant. But in other periods no difference was noted.

\section{CONCLUSION}

The treatment responses across the two arms were compared. In arm A, 24 patients $(80 \%)$ showed complete response and in arm B, 20 patients (66.7\%) showed complete response; partial responses were 2 $(6.7 \%)$ and $4(13.3 \%)$ in the two arms respectively. Progressive disease was noticed in 2 patients $(6.7 \%)$ in each arm. Two patients (6.7\%) in arm A and 3 patients $(10 \%)$ in arm B came back with recurrence. One death $(3.3 \%)$ was reported in conventional group i.e. in arm B. Clinically this difference warrants much attention though statistical significance was not established in this regard.

\section{REFERENCES}

1. Wen PY, Kesari S. Malignant Gliomas in Adult. NEJM, 2008; 3(5):492-7.

2. Ries LAG, Eisner MP, Kosary CL (eds). SEER Cancer Statistics, 1973-1998. Bethesda, MD, National Cancer Institute, 2001.

3. Mehta M, Michael A, Vogelbaum, Chang S, Patel N. Neoplasms of the central nervous system, in De Vita V Jr, Hellman S, Rosenberg S (eds): Cancer: Principales and Practice of Oncology. Philadelphia, PA, Lippincott Williams and Wilkins, 2011:1700-1749.

4. Barret A, Dobbs J, Morris S. Central nervous system: Practical Radiationtherapy Planning. Tom Roques, 2008:205-230.

5. Ahmed R, Oborski MJ, Hwang M, Lieberman FS, Mountz JM. Malignant gliomas: current perspectives in diagnosis, treatment, and early response assessment using advanced quantitative imaging methods. Cancer management and research. 2014;6:149.

6. Melean G, Sestini R, Ammannati F, Papi L. Genetic insights into familial tumors of the nervous system. InAmerican Journal of Medical Genetics Part C: Seminars in Medical Genetics 2004 Aug 15 (Vol. 129, No. 1, pp. 74-84). Hoboken: Wiley Subscription Services, Inc., A Wiley Company.

7. Peter ID, Linet MS, Heineman EF. Etiology of brain tumors in adults. Epidemiologic reviews. 1995 Jan 1;17(2):382-414. 
8. Curran Jr WJ, Scott CB, Horton J, Nelson JS, Weinstein AS, Fischbach AJ, Chang CH, Rotman M, Asbell SO, Krisch RE, Nelson DF. Recursive partitioning analysis of prognostic factors in three Radiation Therapy Oncology Group malignant glioma trials. JNCI: Journal of the National Cancer Institute. 1993 May 5;85(9):704-10.

9. Friedman HS, McLendon RE, Kerby T, Dugan M, Bigner SH, Henry AJ, Ashley DM, Krischer J, Lovell S, Rasheed K, Marchev F. DNA Mismatch Repair and $\mathrm{O}^{\wedge}$ 6-Alkylguanine. DNA Alkyltransferase Analysis and Response to Temodal in Newly Diagnosed Malignant Glioma. Journal of Clinical Oncology. 1998 Dec 1;16(12):3851-7.

10. Yung WA, Albright RE, Olson J, Fredericks R, Fink K, Prados MD, Brada M, Spence A, Hohl RJ, Shapiro W, Glantz M. A phase II study of temozolomide vs. procarbazine in patients with glioblastoma multiforme at first relapse. British journal of cancer. 2000 Sep;83(5):588-93.

11. Choi JW, Lee MM, Kim IA, Kim JH, Choe G, Kim CY. The outcomes of concomitant chemoradiotherapy followed by adjuvant chemotherapy with temozolomide for newly diagnosed high grade gliomas: the preliminary results of single center prospective study. Journal of Korean Neurosurgical Society. 2008 Oct;44(4):222-227.

12. Walker MD, Alexander E, Hunt WE, MacCarty CS, Mahaley MS, Mealey J, Norrell HA, Owens G, Ransohoff J, Wilson CB, Gehan EA. Evaluation of BCNU and/or radiotherapy in the treatment of anaplastic gliomas: a cooperative clinical trial. Journal of neurosurgery. 1978 Sep 1;49(3):333-43.

13. Mahaley MS, Mettlin C, Natarajan N, Laws ER, Peace BB. National survey of patterns of care for brain-tumor patients. Journal of neurosurgery. 1989 Dec 1;71(6):826-36.

14. Walter MD, Alexander E, Hunt WE. Evaluation of $\mathrm{BCNU}$ and/or radiation therapy in the treatment of anaplastic gliomas. J Neurosurg. 1978;49(333):1978-343.

15. Medical Research Council Brain Tumour Working Party. Randomized trial of procarbazine, lomustine, and vincristine in the adjuvant treatment of high-grade astrocytoma: a Medical Research Council trial. Journal of Clinical Oncology. 2001 Jan 15;19(2):509-18.

16. Sathornsumetee S, Rich JN, Reardon DA. Diagnosis and treatment of high-grade astrocytoma. Neurologic clinics. 2007 Nov $1 ; 25(4): 1111-39$. 